\title{
A combination of multimodal physical exercises in real and virtual environments for individuals after chronic stroke: study protocol for a randomized controlled trial
}

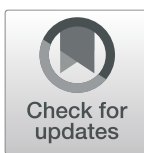

\author{
Natalia Araujo Mazzini ${ }^{1}$ D, Murilo Groschitz Ruas Almeida', José Eduardo Pompeu², Janaine Cunha Polese ${ }^{3}$ and \\ Camila Torriani-Pasin ${ }^{1 *}$
}

\begin{abstract}
Background: Multimodal physical exercises already have well-established benefits for the post-stroke population that influence gait functional capacity, balance, gait, cognition, and quality of life. This type of intervention can be performed in both real and virtual environments. Considering the characteristics of both environments, it is questioned to what extent the combination of interventions in real and virtual environments could result in improvement in post-stroke impairments.

Methods/design: We will conduct a randomized clinical trial with three groups: a real multimodal group (RMG), a virtual multimodal group (VMG), and a combined multimodal group (CMG). It was estimated that we will need a sample of 36 participants (12 per group). RMG individuals will only perform multimodal physical exercises in a real environment two times per week for 60 min per session for 15 weeks. VMG individuals will perform exercises of the same duration over the same time frame but only in a virtual environment. CMG individuals will hold a weekly session in a real environment and another weekly session in virtual environment. The primary outcome measure will be healthrelated quality of life, evaluated using the Stroke Impact Scale; effects on cognition (Montreal Cognitive Assessment), balance (Berg Balance Scale), mobility (Timed Up \& Go), self-selected gait speed (10-meter walk test), and gait functional capacity (6-min walk test) will be investigated as secondary outcome measures. Participants will be evaluated before the beginning of the intervention, immediately after the end of the intervention, and at 1-month follow-up without exercise. If the data meet the assumptions of the parametric analysis, the results will be evaluated by analysis of variance $(3 \times 3)$ for the group factor, with repeated measures while taking into account the time factor. The post hoc Tukey test will be used to detect differences $(a=0.05)$.
\end{abstract}

Discussion: This study represents the first clinical trial to include three groups considering physical exercise in real and virtual environments, isolated and combined, that counterbalances the intensity and volume of training in all groups. This study also includes a control of progression in all groups along the 15-week intervention. The outcome measures are innovative because, according to International Classification of Functioning, Disability and Health, activity and participation are the targets for effectiveness evaluation.

Trial registration: Combinação de exercícios físicos multimodais em ambientes real e virtual para indivíduos pós acidente vascular cerebral crônico, RBR-4pt72m. Registered on 29 August 2016.

Keywords: Stroke, Physical exercises, Virtual reality, Quality of life.

\footnotetext{
* Correspondence: camilatorriani@gmail.com

${ }^{1}$ Motor Behavior Laboratory, School of Physical Education and Sport,

University of São Paulo, São Paulo, SP, Brazil

Full list of author information is available at the end of the article
}

(c) The Author(s). 2019 Open Access This article is distributed under the terms of the Creative Commons Attribution 4.0 International License (http://creativecommons.org/licenses/by/4.0/), which permits unrestricted use, distribution, and reproduction in any medium, provided you give appropriate credit to the original author(s) and the source, provide a link to the Creative Commons license, and indicate if changes were made. The Creative Commons Public Domain Dedication waiver (http://creativecommons.org/publicdomain/zero/1.0/) applies to the data made available in this article, unless otherwise stated. 


\section{Background}

The diverse impairments observed after a stroke, associated with the reduction of intrinsic motivation and the presence of preexisting or acquired comorbidities, lead to a vicious cycle of decreased activity and increased exercise intolerance. As a consequence, secondary complications, such as reduced cardiorespiratory fitness, muscle atrophy, osteoporosis, and circulation impairment in the lower extremities, may occur and generate greater dependence in the activities of daily living and impact the social interactions of these individuals [1].

Different modalities of physical exercises already have well-established benefits for individuals after chronic stroke, including repercussions for cardiovascular capacity [2], muscle strength $[3,4]$, balance $[5,6]$, gait $[7,8]$, and cognition [9]. In order to maximize the effects of the exercises, there is a tendency to investigate the effects of multimodal protocols. According to Saunders et al. [10], a multimodal protocol refers to interventions based on the combination of physical exercises of different components, such as cardiorespiratory, muscular strength, and flexibility.

Multimodal physical exercises can be performed in both real and virtual reality environments. The interventions performed in real environments are the most commonly used in the clinical context. Characteristics of interventions performed in real environments include a high interactive relationship between the professional and the patient, high ecological validity, the possibility of individual or group applications, not requiring technological resources, and the ability to be applied in the home according to each patient's needs.

Conversely, virtual reality-based interventions present features such as an environment rich in visual and auditory information with immediate and multisensory feedback [11], real-time simulation of tasks or environments, three-dimensional interactive and immersive experiences, a computerized interface, active and safe patient participation [12], and the ability to provide information with an external focus of attention [13, 14]. In a systematic review, Laver et al. [15] found that the addition of virtual reality to conventional methods resulted in improved upper limb function. However, they also found insufficient evidence regarding the superiority of virtual reality for promoting walking speed and balance. They were unable to pool results related to cognition, improvement of social participation, and health-related quality of life (HRQoL) because few studies included assessments of cognition and HRQoL to achieve meta-analysis requirements for these outcomes [15]. Therefore, these parameters should be investigated in future studies; in addition, the authors also emphasized the need for training lasting longer than $15 \mathrm{~h}$ of intervention and that future studies should set the number of participants screened for eligibility criteria.

Considering the characteristics of both environments, it is questioned to what extent the combination of interventions in the real and virtual environments could result in improvement in post-stroke impairments. There are few studies that have sought to find answers to this question. In the Shin et al. [16] study, the control group performed $1 \mathrm{~h}$ of occupational therapy per session, and the experimental group performed $30 \mathrm{~min}$ of occupational therapy plus $30 \mathrm{~min}$ of virtual reality. The results showed positive effects in both groups, except for the domain related to the limitations due to physical problems measured by the Short Form Health Survey scores, in which experimental group (EG) obtained greater benefits. Rajaratnam et al. [17] found positive results for balance and mobility measurements for the group that performed $40 \mathrm{~min}$ of conventional therapy plus $20 \mathrm{~min}$ of self-directed virtual reality balance training per session, compared with the control group, which performed 60 min of conventional therapy.

Saposnik and Levin [18] claimed there were few publications regarding the combination of multimodal physical exercises in real and virtual environments. Most of the existing studies did not investigate long-term effects, including follow-up, and added intervention time to the experimental groups, which provided them with an advantage in the total intervention time received. In addition, there is an important diversity in the literature regarding the profile characteristics of individuals with stroke, considering acute, subacute, and chronic patients. Thus, the results found in the previous studies [19-24] do not allow consistent conclusions to be made about the effects of the combination of multimodal exercises in real and virtual environments in individuals after chronic stroke.

This study seeks to answer whether the combination of multimodal physical exercises in real and virtual environments could bring additional benefits to the quality of life, cognition, gait, and balance of individuals after chronic stroke. We also intend to clarify the effects of interventions with multimodal physical exercises when performed only in a real environment or only in a virtual environment and to investigate whether the possible effects remain after 1 month without participating in physical exercises.

This study aims to investigate the effects of a protocol of multimodal physical exercises in real and virtual environments for individuals who have survived a stroke.

\section{Methods/design}

\section{Trial design}

This is a prospective, randomized, single-blind trial of a 15-week exercise program to investigate the effects of the combination of physical exercise in real and virtual 
environments for chronic post-stroke individuals. It was approved by the Ethics and Research Committee on $\mathrm{Hu}$ man Beings of the School of Physical Education and Sports at the University of São Paulo (CAE no. 40,688, 114.9.0000.5391) and is registered with ensaiosclinicos. gov (no. RBR-4pt72m).

All participants and/or their relatives will provide written informed consent prior to participant enrollment. A Consolidated Standards of Reporting Trials (CONSORT) flow diagram of the trial is shown in Fig. 1, and a Standard Protocol Items: Recommendations for Interventional Trials (SPIRIT) checklist is provided in Additional file 1.

\section{Study setting}

A prospective, randomized trial with concealed allocation, blinded assessors, and intention-to-treat analysis will be carried out. The study will be conducted in the Motor Behavior Laboratory in the School of Physical Education and Sports at the University of Sao Paulo, Brazil.

\section{Eligibility screening}

Participants will be recruited from the waiting list for the rehabilitation program at the Physical Education and Sports School in Sao Paulo, Brazil, and will be screened for eligibility by neurological rehabilitation physiotherapy specialists.

\section{Inclusion and exclusion criteria}

Screening will be done to obtain personal data for the individuals (name, sex, date of birth, address, telephone, and schooling), medical history, brain injury topography, and type of locomotion. After this initial screening, the individuals will be referred for a specific medical evaluation in order to investigate their health conditions, request the necessary tests, and follow the medicines used. Only those individuals who receive medical release for the practice of physical exercises will enter the study.

The inclusion criteria will be chronic phase stroke (more than 6 months post-stroke, according to Bernhardt et al. [25]), territory of the lesion in the middle cerebral

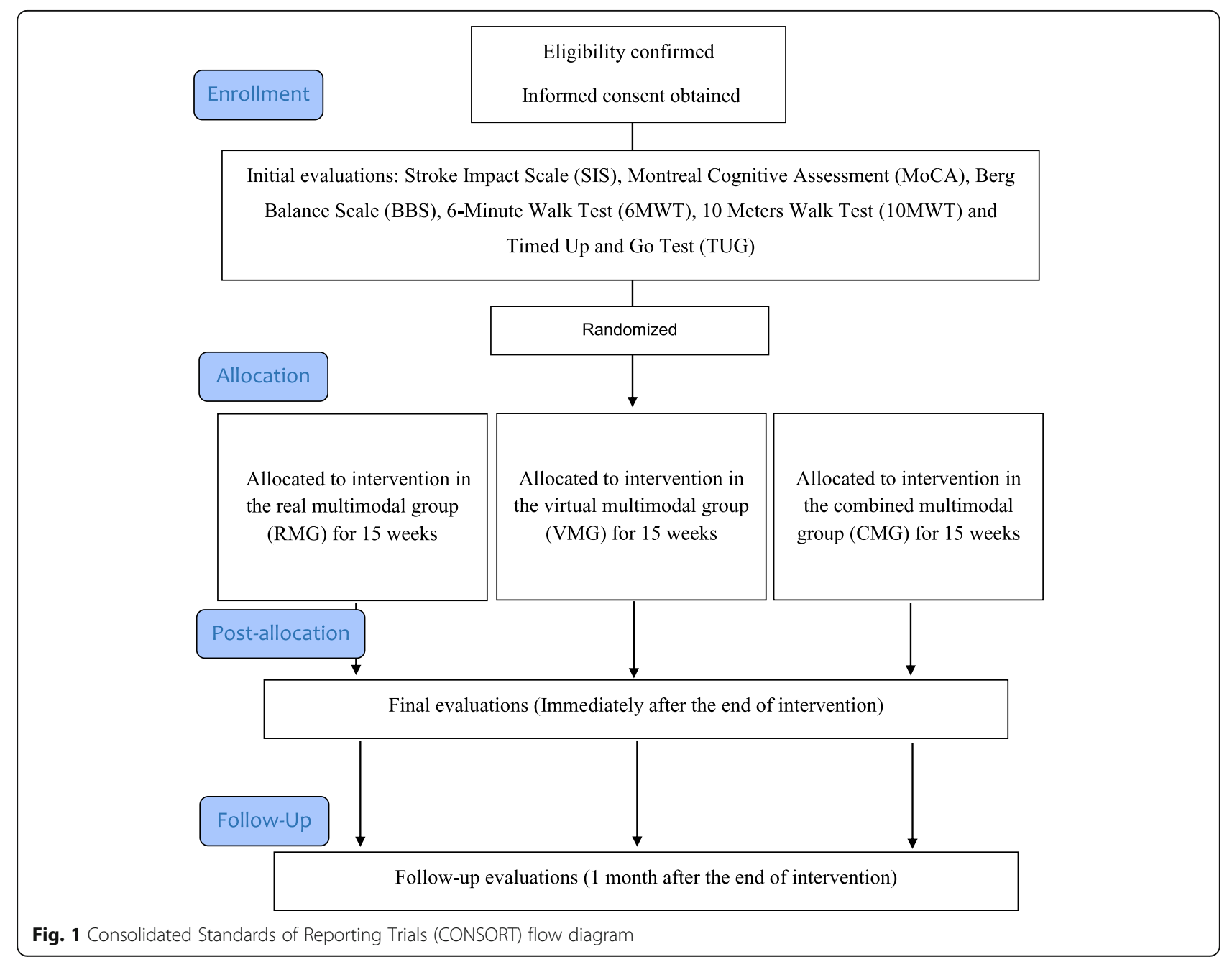


artery or anterior cerebral artery, both types of stroke (ischemic and hemorrhagic), cognition greater than 24 points on the Mini Mental State Examination, at least 2 months of noninvolvement in other structured forms of physical exercise intervention (community), and no experience in virtual reality games.

The exclusion criteria will be individuals with any type of cardiovascular complication that would contraindicate physical exercise, individuals who underwent surgeries to attenuate clinical conditions resulting from stroke, and those who have undergone chemical blockade to reduce spasticity.

\section{Informed consent}

Individuals who fit the study requirements will receive the informed consent form, which will be duly read and explained by the researcher and signed by the individuals and/or their legal guardians.

\section{Randomization procedures}

Participants who meet the study inclusion criteria will be randomized into one of three sample groups-the real multimodal group (RMG), the virtual multimodal group (VMG), or the combined multimodal group (CMG) - through a draw of numbered (generated by computer) and sealed opaque envelopes. The evaluators have no knowledge of the results of the individual allocation (blinded evaluators). However, the same is impossible to perform with the professionals who will perform the interventions; because the interventions are supervised and progressive, there are clearly distinct protocols between groups of individuals.

To characterize the individuals, the Mini Mental State Examination, Fugl-Meyer Assessment, and Orpington Prognostic Scale will be applied. The individuals will also be characterized by the demographic variables of sex, age, type of stroke, injury time, affected brain hemisphere, and schooling.

\section{Outcome measures and outcome assessment}

The primary outcome will be the domain "activities of daily living" from the Stroke Impact Scale (SIS). This will be used to measure the individuals' levels of participation. As secondary outcome measures, the other domains from the SIS will be used, as well as their total score and the percentage of recovery from stroke, for the purpose of measuring the range of structures/functions and activities. These will be measured using the Montreal Cognitive Assessment (MoCA), Berg Balance Scale (BBS), 6-minute walk test (6MWT), 10-meter walk test (10MWT), and Timed Up \& Go Test (TUG).

All measurements will be carried out by trained evaluators who will not be involved in the interventions and will be blinded to the allocation of individuals to the groups. All individuals will be evaluated at three moments: before the beginning of the interventions (baseline), immediately after the end of the interventions (posttest), and 1 month after the end of the interventions (follow-up). The schedule of enrollment, allocation, and postallocation is provided in Table 1.

\section{Intervention}

The intervention for the three sample groups will be 15 weeks long and will consist of two weekly sessions of 60 min each. All interventions will be carried out at the School of Physical Education and Sports of the University of Sao Paulo, Brazil.

The individuals allocated to RMG will carry out sessions in multimodal training in a real environment. The sessions will be divided into four moments, based on guidelines from Billinger et al. [26]: balance and cognition (15 $\mathrm{min}$ in duration), training of the aerobic

Table 1 Outcomes assessment time-points and instruments

\begin{tabular}{|c|c|c|c|c|c|c|}
\hline & \multirow[t]{2}{*}{ Instruments/methods } & \multirow[t]{2}{*}{ Enrolment } & \multirow[t]{2}{*}{ Baseline } & \multirow[t]{2}{*}{ Allocation } & \multicolumn{2}{|c|}{ Post allocation } \\
\hline & & & & & $\begin{array}{l}\text { Post test } 15 \\
\text { weeks }\end{array}$ & $\begin{array}{l}\text { Follow up } \\
19 \text { weeks }\end{array}$ \\
\hline $\begin{array}{l}\text { Eligibility screen: Socio- } \\
\text { demographic variables }\end{array}$ & $\begin{array}{l}\text { Structured interview, recording age, brain injury, } \\
\text { schooling and medical history }\end{array}$ & $x$ & & & & \\
\hline Informed consent & Informed Consent Term & $x$ & & & & \\
\hline Allocation & 3 intervention groups: RMG, VMG or CMG & & & $x$ & & \\
\hline Quality of life & SIS & & $x$ & & $x$ & $x$ \\
\hline Cognitive & MOCA & & $x$ & & $x$ & $x$ \\
\hline Gait Functional Capacity & $6 \mathrm{MWT}$ & & $x$ & & $x$ & $x$ \\
\hline Self-selected gait speed & $10 \mathrm{MWT}$ & & $x$ & & $x$ & $x$ \\
\hline Mobility & TUG & & $x$ & & $x$ & $x$ \\
\hline Balance & Berg Balance Scale (BBS) & & $x$ & & $x$ & $x$ \\
\hline
\end{tabular}


component and muscular strength (20 min each), and a fourth moment focused on flexibility and relaxation (5 min in duration). Aerobic circuits, games, adapted sports, strength training stations, tasks with varying bases of support, double tasks, memorization tasks, and other strategies will be used. Materials will include balls of diverse sizes and constitutions, dumbbells, washers, cones, hula hoops, bladders, wooden sticks, mats, shuttlecocks, and rackets.

Individuals assigned to the VMG group will carry out individualized multimodal training sessions in a virtual environment. For this protocol, there will be eight games of Stability and Balance Learning Environment, a system of virtual reality developed by Motekforce Medical (Amsterdam, the Netherlands), which is composed of a $10.76-\mathrm{ft}^{2}$ force platform, three projectors, six infrared cameras, a sound system, and a touchscreen panel for selection and control of variables. The games of this virtual reality device were produced and adapted to be beneficial for special populations that have balance and movement disorders, such as people with neurological disorders (stroke, cerebral palsy, Parkinson's disease, traumatic brain injury), orthopedic disorders (amputees, osteoarthritis, musculoskeletal disorders), and the geriatric population (elderly with increased risk of falling). Stability and Balance Learning Environment requires the player to move the entire body, which includes tasks of reach, stationary gait, and others.

These games were grouped into two blocks of sessions that will be applied alternately throughout the intervention. The odd sessions (Fig. 2) will include the games balloon pop, city ride, hit the mole, and $2 \mathrm{~d}$ maze, and the even sessions (Fig. 3) will consist of the games road encounters, road stepping, paper flight, and hit knees. The number of selected games as the group session in two models aims to make the most motivating interventions possible and keep the characteristics between the games of both models similar.

In order to guarantee the multimodal character of the virtual intervention and to make it similar to the real intervention, the games were classified according to the perceptual and motor demands of the virtual reality systems protocol developed by Cairolli et al. [27]. The use of this classification protocol ensured that the games placed similar demands on the individuals. For example, the demand for strength and cardiovascular resistance could be consistent across the selected games.

Finally, individuals in the CMG group will perform an intervention that combines the protocols of the two other groups described above. In this way, one of the weekly sessions will be held in a real environment and the other in a virtual environment.
Before the start of all sessions, regardless of the group, the individuals' blood pressure will be verified in order to ensure that no one initiates the practice of physical exercise with a blood pressure greater than 160/105 $\mathrm{mmHg}$ [28]. This contributes to the safety of the practice. To register and control the data, stethoscopes (Efficacy line, Bunzl Saúde, Brazil) and manual blood pressure cuff aneroid sphygmomanometers (Solidor, Bunzl Saúde, Brazil) will be used.

Aerobic exercise intensity control will be performed to ensure the safety of the interventions in the three groups. During the interventions, the individuals' heart rate (HR) will be monitored constantly in order to keep it aligned with the range prescribed individually using the Karvonen formula $\left(\left(\mathrm{HR}_{\text {maximum }}-\mathrm{HR}_{\text {rest }}\right) \times\right.$ percentage $\mathrm{HR}$ intensity $\left.+\mathrm{HR}_{\text {rest }}\right)$ [29]. We will use $40 \%$ and $70 \%$ of the reserve $\mathrm{HR}$, as determined by Billinger et al. [26], as the lower and upper intensity thresholds, respectively. The maximum HR will be calculated using the formula 220 - age in years [30], except in individuals who use beta-blockers. Thus, the maximum HR will be calculated by the formula $164-0.7 \times$ age in years [31]. For the control of HR ranges, we will use Polar brand $\mathrm{H} 7$ frequency meters synchronized with the Polar Team application (Polar, Bethpage, NY, USA). As a complementary form to intensity control, we will use the Borg Rating of Perceived Exertion [32, 33], which correlates with the objective measures of the workload level and HR [24]. The individuals' perception of effort will be verified before the beginning of each session and soon after the onset of the aerobic component in the real environments, or soon after the practice of each of the games in the virtual environment sessions. We will adjust the offer of stimuli that indicate perceptions between values 11 and 14, as determined by Billinger et al. [26].

Resistance exercise intensity will also be controlled according to Billinger et al. [26]. All individuals will perform eight to ten exercises, preferably involving the major muscle groups. Each exercise will include 1 to 3 sets of 10 to 15 repetitions.

Every 5 weeks, the aerobic and resistance exercise intensity will be adjusted in terms of progression. By the end of the 15-week intervention period, we will have performed two progressions. The aerobic component will include gradual increases $(10 \%)$ of the HR percentage, and the resistance component will increase through larger numbers of sets or repetitions for each exercise.

\section{Measurements}

Quality of life will be evaluated through the SIS. This scale evaluates quality of life by measuring 59 items 


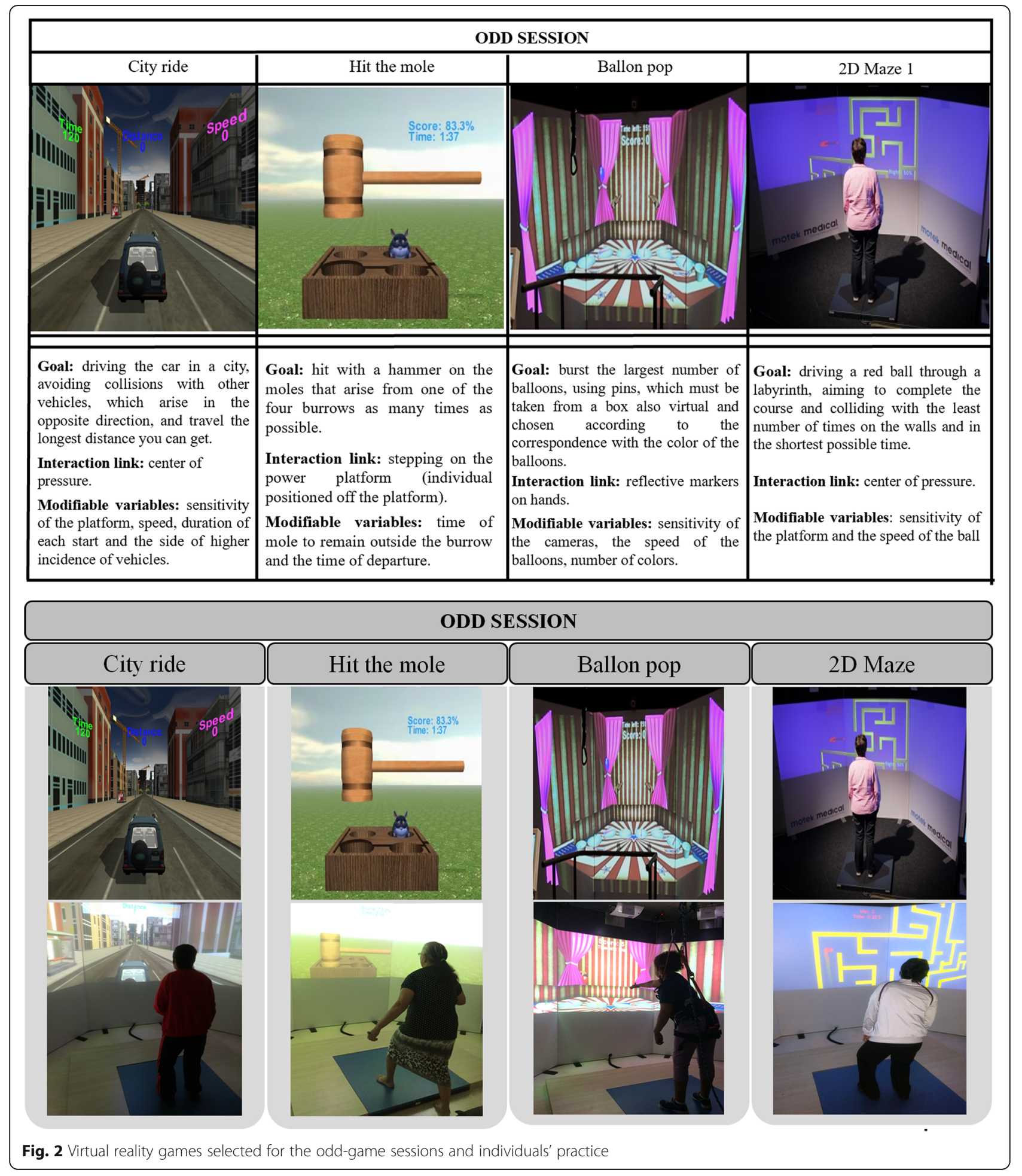

categorized in 8 domains: strength (4 items), hand function (5 items), activities of daily living/instrumental activity of daily living (10 items), mobility (9 items), communication items [7], emotion (9 items), memory and reasoning (7 items), and participation function (8 items). Each item is rated on a 5-point Likert scale in terms of difficulty the patient has experienced in completing each item. Summative scores will be generated for each domain, ranging from 0 to 100 . An extra question will be asked in order to know, on a scale from 0 to 

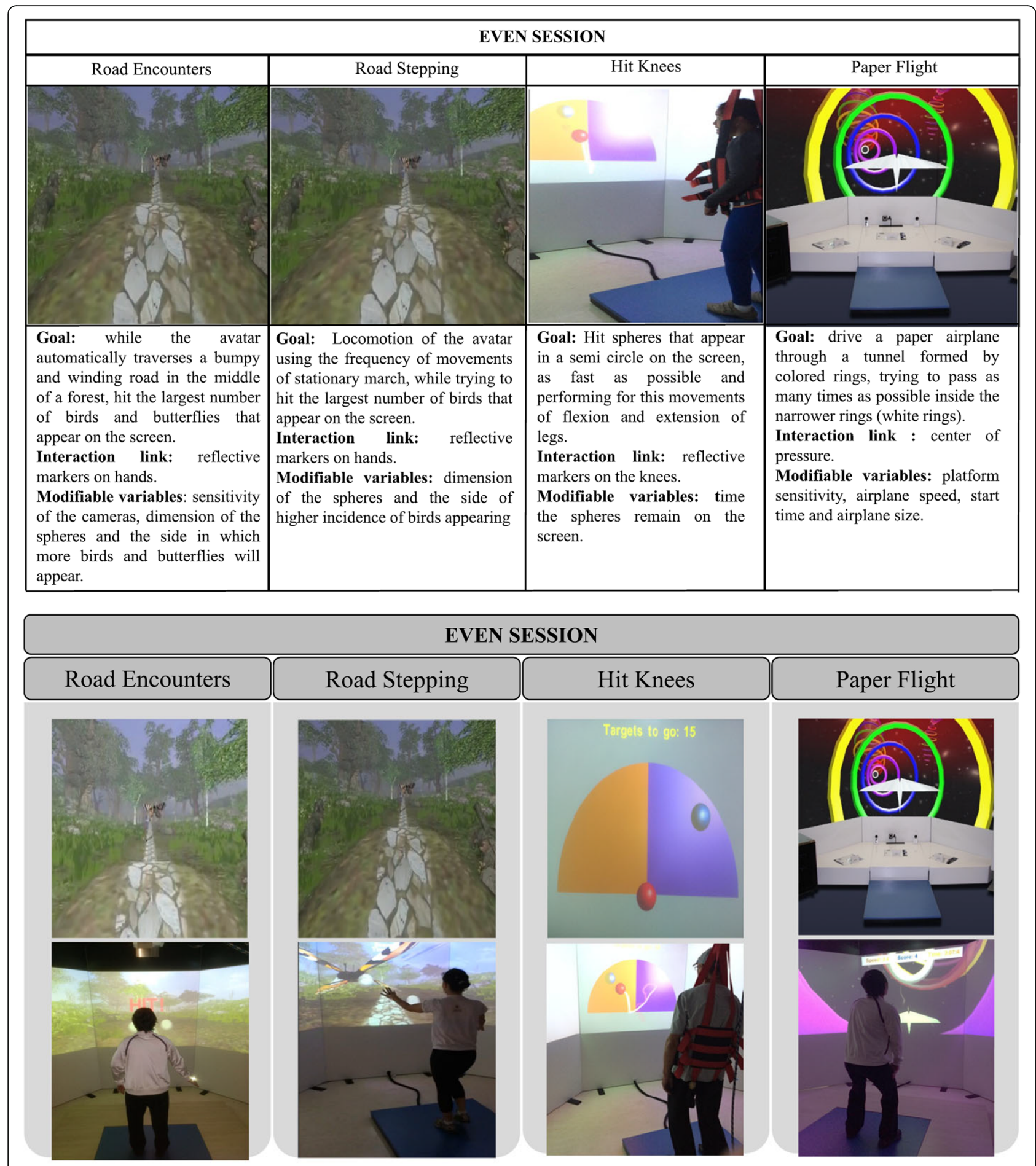

Fig. 3 Virtual reality games selected for the even-game sessions and individuals' practice

100, how much the individual feels they have recovered since their stroke. Our primary outcome measure will be the domain of the activities of daily living/instrumental activity of daily living, and the rest of the domains will be used as secondary outcome measures [34]. The
Brazilian version of SIS 3.0 has satisfactory internal consistency, test-retest reliability, convergent validity, and discriminant validity in stroke patients [34]. SIS 3.0 is a specific measure of psychometrically robust HRQoL that can be useful in assessing the consequences of 
stroke in different cultural contexts [34]. For some of the domains that make up this scale, there are values of minimum detectable change (MDC) and clinically important difference (CID). According to Lin et al. [35], the values are strength (MDC 24.0 and CID 9.2), activities of daily living/instrumental activities of daily living (MDC 17.3 and CID 5.9), mobility (MDC 15.1 and CID 4.5), and hand function (MDC 25.9 and CID17.8).

Cognition will be evaluated through the MoCA. MoCA was developed as an instrument of rapid screening for mild cognitive dysfunction and for evaluating different cognitive domains, such as attention and concentration, executive functions, memory, language, constructional visual skills, conceptual thinking, calculations, and logical reasoning. The scale ranges from 0 to 30 [36]. In the chronic phase of stroke, MoCA has a good correlation with other short cognitive tests and shows high sensitivity and specificity in the prediction of post-stroke cognitive deterioration [37]. There is no consensus on the cutoff to define cognitive impairment for stroke, but most authors use a 26-point cutoff. There are no MDC values for this scale [38]. For the subacute phase of stroke, the scale shows excellent internal consistency $(\alpha=0.78)$ [39].

Balance will be evaluated through the BBS, which organizes the quantitative description (classification of 0 to 4) of functional balance ability into 14 items common to daily life. It has a minimum score of 0 and a maximum of 56 [40]. The measurement's MDC is 4.66 [41], and it has excellent test-retest reliability (intraclass correlation $[\mathrm{ICC}]=0.98)[42]$.

Gait functional capacity will be evaluated through the 6MWT, which quantifies the maximum distance, expressed in meters, that the individual is able to walk in $6 \mathrm{~min} \mathrm{[43].} \mathrm{The} \mathrm{measurement's} \mathrm{MDC} \mathrm{is} 36.6 \mathrm{~m} \mathrm{[44];}$ the CID is $34.4 \mathrm{~m}$ [45]; and it has excellent test-retest reliability $(\mathrm{ICC}=0.99)$ [44].

Self-selected gait speed will be evaluated through the 10MWT, which determines the average speed, expressed in meters per second, that the individual applies for a distance of $10 \mathrm{~m}$. The measurement CID is $0.06 \mathrm{~m} / \mathrm{s}$ [46], and it has excellent test-retest reliability $(\mathrm{ICC}=0.94) \quad[44]$.

Mobility will be evaluated through the TUG, which determines the time, expressed in seconds, that the individual takes to perform a task involving a change of direction, transfer, and gait [47]. The measurement's MDC is $2.9 \mathrm{~s}$, and it has excellent test-retest reliability $($ ICC $=0.96)$ [44].

\section{Statistical analysis}

It was estimated that a sample of 36 participants (12 per group) would provide $88 \%$ power $(\alpha=5 \%)$ to detect a difference between group means of 17.3 (MDC of the activities of daily living/instrumental activities of daily living domain of the SIS) using the program G*Power 3 [48], which takes into account the number of sample groups and the number of evaluation measures. To reach this total number of individuals, at least 43 individuals must be recruited, allowing a dropout rate of $20 \%$.

For the mathematical treatment and statistical analysis of the data, Excel software (Microsoft, Redmond, WA, USA) will be used for the tabulation of data, and SPSS version 13 software (SPSS, Chicago, IL, USA) will be used for statistical analyses. A significance level of $5 \%$ will be adopted. Initially, for the characterization of the sample, descriptive analysis and the $t$ test will be used to examine differences between the RMG, VMG, and CMG groups. Subsequently, for the analysis of the normality and homogeneity of the results of the primary outcome measure and secondary outcomes, the Shapiro-Wilk and Levene tests will be used, respectively. After observing the assumptions for normality and homogeneity, parametric analysis will be performed using twoway analysis of variance ( 3 groups by 3 moments [baseline, post-test, and follow-up]) and post hoc Tukey test. The mean between-group difference and 95\% confidence intervals will be reported for all outcomes. If any subject gives up the protocol, their measured outcomes will be analyzed on an intentionto-treat basis.

\section{Discussion}

The proposed clinical trial is of high clinical significance to the field of neurological rehabilitation and to stroke in particular. Physical exercises are an important intervention strategy to promote improvement of gait functional capacity, muscle strength, balance, gait, and cognition in individuals after stroke [9]. In addition, physical exercises improve the quality of life for the post-stroke population. Billinger et al. [26] argued that physical exercise modalities should complement each other to provide a more integrated form of intervention. Thus, multimodal physical exercise protocols must be implemented for this population.

However, the effects of multimodal physical exercises when performed in different environments are not well established in the literature, and the effects of the combination of real and virtual environments are not known. Thus, the findings of this clinical trial may clarify such shortcomings, especially regarding outcomes related to social participation and activity, components of the International Classification of 
Functioning, Disability and Health that have been poorly investigated.

Currently, healthcare professionals are advised to offer broad, nonspecific recommendations regarding exercise. This new information on the effectiveness of physical exercise in a real or virtual environment will allow health professionals to make evidence-based treatment recommendations for the best environment in which patients can carry out their physical exercise prescription.

The design of the proposed study has strong points, such as the presence of three sample groups that are similar in frequency, intensity, and volume of training; a period of intervention longer than that usually found in currently published studies; an assessment of outcomes in follow-up; and an analysis of evaluation measures in the context of social participation, one of the domains of the International Classification of Functioning, Disability and Health.

In conclusion, this study represents the first clinical trial to include three groups while considering the prescription of physical exercise in real and virtual environments, both isolated and combined, that counterbalances the intensity and volume of the training in all groups. This study also includes the control of progression in all groups during the 15-week intervention. Although very revealing to evaluate the effectiveness of an intervention, measures evaluating the domains of activity and participation are rarely investigated in the literature. Thus, our study is innovative because it includes these measures, differing from the vast majority of studies, which evaluate only aspects related to body structures and functions.

In addition, after the data analysis, we intend to perform a cost-benefit review, based on Lloréns et al. [49]. This analysis will include human and material resources as well as professional-patient relationships. This analysis will include the comparison between our study and that of Saposnik et al. [50], which showed low-cost and easy-to-access interventions being as useful as the least accessible and least costeffective.

\section{Trial status}

This study protocol is in progress and is not yet related to requests.

Date recruitment began on August 30, 2016.

Approximate date when recruitment will be completed: first half of 2019.

Date of protocol registration: August 25, 2016.

Protocol version number: RBR-4pt72m. Proof review: 27th May 2019.

\section{Additional file}

Additional file 1: SPIRIT 2013 checklist: recommended items to address in a clinical trial protocol and related documents. (PDF $116 \mathrm{mb}$ )

\section{Abbreviations \\ 10MWT: 10-Meter walk test; 6MWT: 6-Min walk test; BBS: Berg Balance Scale; CID: Clinically important difference; CMG: Combined multimodal group; HR: Heart rate; HRQoL: Health-related quality of life; ICC: Intraclass correlation; MDC: Minimum detectable change; MoCA: Montreal Cognitive Assessment; RMG: Real multimodal group; SIS: Stroke Impact Scale; TUG: Timed Up \& Go Test; VMG: Virtual multimodal group}

\section{Authors' contributions}

All chief investigators contributed to the design of this trial protocol and will be included as authors in final reports and publications of the trial. TBdF will be responsible for the evaluation of the individuals and for the interpretation of the results. NAM and MGRA will be responsible for the implementation of interventions and project writing. JEP and JCP will be responsible for collaborating on the study design, statistical analysis, and results. CTP will be responsible for the study concept, manuscript writing, data analysis, and guiding and directing the project. All authors read and approved the final manuscript.

\section{Funding}

This study was supported by Coordenação de Aperfeiçoamento de Pessoal de Nível Superior (CAPES-PROEX).

Availability of data and materials

The datasets used and/or analyzed during the current study are available from the corresponding author on reasonable request.

\section{Ethics approval and consent to participate}

The study was approved by the Ethics and Research Committee on Human Beings of the School of Physical Education and Sports at the University of São Paulo (CAE no. 406881 14.9.0000.5391) and is registered with ensaiosclinicos.gov (no. RBR-4pt72m). Written and oral informed consent is obtained from all participants prior to their enrollment in the study.

\section{Consent for publication}

We have gained informed consent for publication of the dataset from patients at the point of recruitment to the trial. All the patient details will be fully anonymized.

\section{Competing interests}

The authors declare that they have no competing interests.

\section{Author details}

${ }^{1}$ Motor Behavior Laboratory, School of Physical Education and Sport, University of São Paulo, São Paulo, SP, Brazil. 'Laboratory of Studies in Technology, Functionality and Aging of the Department of Physical Therapy, Speech and Occupational Therapy, School of Medicine, University of São Paulo, São Paulo, SP, Brazil. ${ }^{3}$ Department of Physical Therapy, Medical Sciences College of Minas Gerais, Belo Horizonte, MG, Brazil.

Received: 26 November 2018 Accepted: 6 May 2019

Published online: 16 July 2019

\section{References}

1. Gordon NF, Gulanick M, Costa F, Fletcher G, Franklin BA, Roth EJ, et al. Physical activity and exercise recommendations for stroke survivors: an American Heart Association scientific statement from the Council on Clinical Cardiology, Subcommittee on Exercise, Cardiac Rehabilitation, and Prevention; the Council on Cardiovascular Nursing; the Council on Nutrition, Physical Activity, and Metabolism; and the Stroke Council. Circulation. 2004; 109(16):2031-41.

2. Saltychev M, Sjögren T, Bärlund E, Laimi K, Paltamaa J. Do aerobic exercises really improve aerobic capacity of stroke survivors? A systematic review and meta-analysis. Eur J Phys Rehabil Med. 2016;52(2):233-43. 
3. Lee NK, Kwon JW, Son SM, Kang KW, Kim K, Hyun-Nam S. The effects of closed and open kinetic chain exercises on lower limb muscle activity and balance in stroke survivors. NeuroRehabilitation. 2013;33(1): 177-83.

4. Ouellette MM, LeBrasseur NK, Bean JF, Phillips E, Stein J, Frontera WR, et al. High-intensity resistance training improves muscle strength, self-reported function, and disability in long-term stroke survivors. Stroke. 2004;35(6): 1404-9.

5. Shin WS, Lee SW, Lee YW, Choi SB, Song CH. Effects of combined exercise training on balance of hemiplegic stroke patients. J Phys Ther Sci. 2011; 23(4):639-43.

6. Son SM, Park MK, Lee NK. Influence of resistance exercise training to strengthen muscles across multiple joints of the lower limbs on dynamic balance functions of stroke patients. J Phys Ther Sci. 2014; 26(8):1267-9.

7. Mudge S, Barber PA, Stott NS. Circuit-based rehabilitation improves gait endurance but not usual walking activity in chronic stroke: a randomized controlled trial. Arch Phys Med Rehabil. 2009;90(12): 1989-96.

8. Yang $H C$, Lee $C L$, Lin $R$, Hsu MJ, Chen $C H$, Lin JH, et al. Effect of biofeedback cycling training on functional recovery and walking ability of lower extremity in patients with stroke. Kaohsiung J Med Sci. 2014; 30(1):35-42.

9. Cumming TB, Tyedin K, Churilov L, Morris ME, Bernhardt J. The effect of physical activity on cognitive function after stroke: a systematic review. Int Psychogeriatr. 2012;24(4):557-67.

10. Saunders DH, Sanderson M, Hayes S, Kilrane M, Greig CA, Brazzelli M, et al. Physical fitness training for stroke patients. Cochrane Database Syst Rev. 2016;3(3):CD003316.

11. Burdea GC. Virtual rehabilitation - benefits and challenges. Methods Inf Med. 2003:42(5):519-23.

12. Sveistrup H. Motor rehabilitation using virtual reality. J Neuroeng Rehabil. 2004;1:10.

13. Corrêa AGD, de M Monteiro CB, da Silva TD, de Lima-Alvarez CD, Fichemann IK, Tudella E, et al. Realidade virtual e jogos eletrónicos: uma proposta para deficientes. In: Realidade virtual na paralisia cerebral. Sao Paulo: Plêiade; 2011

14. dos Palma GCS, Freitas TB de F, Bonuzzi GMG, Soares MAA, Leite PHW, Mazzini NA, et al. Effects of virtual reality for stroke individuals based on the International Classification of Functioning and Health: a systematic review. Top Stroke Rehabil. 2016;24(4):269-78.

15. Laver KE, Lange B, George S, Deutsch JE, Saposnik G, Crotty M. Virtual reality for stroke rehabilitation. Cochrane Database Syst Rev. 2017;11: CD008349.

16. Shin JH, Park SB, Jang SH. Effects of game-based virtual reality on healthrelated quality of life in chronic stroke patients: a randomized, controlled study. Comput Biol Med. 2015;63:92-8.

17. Rajaratnam BS, Gui Kaien J, Lee Jialin K, Sweesin K, Sim Fenru S, Enting L, et al. Does the inclusion of virtual reality games within conventional rehabilitation enhance balance retraining after a recent episode of stroke? Rehabil Res Pract. 2013;2013:649561.

18. Saposnik G, Levin M. Virtual reality in stroke rehabilitation: a meta-analysis and implications for clinicians. Stroke. 2011;42(5):1380-6.

19. Chow RTK, Chan ACM, Tong JMC. Effectiveness of virtual reality in balance training in stroke rehabilitation: a pilot study. Hong Kong Physiother J. 2013; 31(2):100.

20. Lee $\mathrm{CH}$, Kim Y, Lee BH. Augmented reality-based postural control training improves gait function in patients with stroke: randomized controlled trial. Hong Kong Physiother J. 2014;32(2):51-7.

21. Morone G, Tramontano M, losa M, Shofany J, lemma A, Musicco M, et al. the efficacy of balance training with video game-based therapy in subacute stroke patients: a randomized controlled trial. Biomed Res Int. 2014;2014 580861

22. Barcala L, Grecco LA, Colella F, Lucareli PR, Salgado AS, Oliveira CS. Visual biofeedback balance training using Wii Fit after stroke: a randomized controlled trial. J Phys Ther Sci. 2013;25(8):1027-32

23. Piron L, Turolla A, Agostini M, Tonin P, Dam M. Motor learning principles for rehabilitation: a pilot randomized controlled study in poststroke patients. Neurorehabil Neural Repair. 2010;24(6):501-8.

24. Yin CW, Sien NY, Ying LA, Chung SF, Tan May Leng D. Virtual reality for upper extremity rehabilitation in early stroke: a pilot randomized controlled trial. Clin Rehabil. 2014;28(11):1107-14.
25. Bernhardt J, Hayward KS, Kwakkel G, Ward NS, Wolf SL, Borschmann K, et al. Agreed definitions and a shared vision for new standards in stroke recovery research: the Stroke Recovery and Rehabilitation Roundtable Taskforce. Neurorehabil Neural Repair. 2017;31(9):793-9.

26. Billinger SA, Arena R, Bernhardt J, Eng JJ, Franklin BA, Johnson CM, et al. Physical activity and exercise recommendations for stroke survivors: a statement for healthcare professionals from the American Heart Association/American Stroke Association. Stroke. 2014;45(8):2532-53.

27. Cairolli FF, Bonuzzi GMG, dos Santos Palma GC, Soares MAA, Pompeu $J E$, de Morais Faria CDC, et al. Development and preliminary research on the measure properties of a perceptual and motor demands assessment protocol for virtual reality systems. Motricidade. 2017;13(1): 50-8.

28. Armstrong L, Balady GJ, Berry MJ, et al. Exercise prescription modifications for cardiac patients. In: Whaley MH, Brubaker PH, Otto RM, editors. ACSM's guidelines for exercise testing and prescription. 7th ed. Philadelphia: Lippincott Williams \& Wilkins; 2006. p. 174-204.

29. Karvonen MJ, Kentala E, Mustala $\mathrm{O}$. The effects of training on heart rate; a longitudinal study. Ann Med Exp Biol Fenn. 1957;35(3):307-15.

30. American College of Sports Medicine. ACSM's guidelines for exercise testing and prescription. 6th ed. Philadelphia: Lippincott Williams \& Wilkins; 2000

31. Brawner CA, Ehrman JK, Schairer JR, Cao JJ, Keteyian SJ. Predicting maximum heart rate among patients with coronary heart disease receiving B-adrenergic blockade therapy. Am Heart J. 2004;148(5):910-4.

32. Borg GAV. Psychophysical bases of perceived exertion. Med Sci Sports Exerc 1982;14(5):377-81.

33. Hampton S, Armstrong G, Shah MV, Li S. Quantification of perceived exertion during isometric force production using Borg scale in healthy individuals and in chronic stroke patients. Top Stroke Rehabil. 2014;21(1):33-9.

34. Carod-Artal FJ, Coral LF, Trizotto DS, Moreira CM. The Stroke Impact Scale 3. 0 : evaluation of acceptability, reliability, and validity of the Brazilian version. Stroke. 2008;39(9):2477-84.

35. Lin K, Fu T, Wu C, Wang Y, Liu J, Hsieh C, et al. Minimal detectable change and clinically important difference of the Stroke Impact Scale in stroke patients. Neurorehabil Neural Repair. 2010;24(5):486-92.

36. Smith T, Gildeh N, Holmes C. The Montreal Cognitive Assessment. Can J Psychiatry. 2007;52(5):329-32.

37. Nasreddine Z, Phillips N, Bédirian V, Charbonneau S, Whitehead V, Colllin I, et al. The Montreal Cognitive Assessment, MoCA: a brief screening tool for mild cognitive impairment. J Am Geriatr Soc. 2005; 53(4):695-9.

38. Chiti G, Pantoni L. Use of Montreal Cognitive Assessment in patients with stroke. Stroke. 2014;45(10):3135-40.

39. Toglia J, Fitzgerald KA, O'Dell MW, Mastrogiovanni AR, Lin CD. The MiniMental State Examination and Montreal Cognitive Assessment in persons with mild subacute stroke: relationship to functional outcome. Arch Phys Med Rehabil. 2011;92(5):792-8.

40. Berg K. Measuring balance in the elderly: development and validation of an instrument. Can J Public Health. 1992;83(August):S7-11.

41. Hiengkaew $V$, Jitaree $K$, Chaiyawat $P$. Minimal detectable changes of the Berg Balance Scale, Fugl-Meyer Assessment Scale, Timed "Up \& Go" test, gait speeds, and 2-minute walk test in individuals with chronic stroke with different degrees of ankle plantarflexor tone. Arch Phys Med Rehabil. 2012; 93(7):1201-8

42. Liston RAL, Brouwer BJ. Reliability and validity of measures obtained from stroke patients using the Balance Master. Arch Phys Med Rehabil. 1996;77(5): 425-30.

43. Rikli RE, Jones CJ. The reliability and validity of a 6-minute walk test as a measure of physical endurance in older adults. J Aging Phys Act. 1998;6: 363-75.

44. Flansbjer UB, Holmbäck AM, Downham D, Patten C, Lexell J. Reliability of gait performance tests in men and women with hemiparesis after stroke. $J$ Rehabil Med. 2005;37(2):75-82.

45. Tang A, Eng J, Rand D. Relationship between perceived and measured changes in walking after stroke. J Neurol Phys Ther. 2012;36(3):115-21.

46. Perera S, Mody SH, Woodman RC, Studenski SA. Meaningful change and responsiveness in common physical performance measures in older adults. J Am Geriatr Soc. 2006;54(5):743-9.

47. Podsiadlo D, Richardson S. The Timed "Up \& Go": a test of basic functional mobility for frail elderly persons. J Am Geriatr Soc. 1991;39(2):142-8. 
48. Faul F, Erdfelder E, Lang AG, Buchner A. G*Power 3: a flexible statistical power analysis program for the social, behavioral, and biomedical sciences. Behav Res Methods. 2007;39(2):175-91.

49. Lloréns R, Noé E, Colomer C, Alcañiz M. Effectiveness, usability, and costbenefit of a virtual reality-based telerehabilitation program for balance recovery after stroke: a randomized controlled trial. Arch Phys Med Rehabil. 2015;96(3):418-25.e2.

50. Saposnik G, Cohen LG, Mamdani M, Pooyania S, Ploughman M, Cheung D, Shaw J, Hall J, Nord P, Dukelow S, Nilanont Y, De los Rios F, Olmos L, Levin M, Teasell R, Cohen A, Thorpe K, Laupacis A, Bayley M. Efficacy and safety of non-immersive virtual reality exercising in stroke rehabilitation (EVREST): a randomised, multicentre, single-blind, controlled trial. Lancet Neurol. 2016; 15(10):1019-27.

\section{Publisher's Note}

Springer Nature remains neutral with regard to jurisdictional claims in published maps and institutional affiliations.

Ready to submit your research? Choose BMC and benefit from:

- fast, convenient online submission

- thorough peer review by experienced researchers in your field

- rapid publication on acceptance

- support for research data, including large and complex data types

- gold Open Access which fosters wider collaboration and increased citations

- maximum visibility for your research: over $100 \mathrm{M}$ website views per year

At $\mathrm{BMC}$, research is always in progress.

Learn more biomedcentral.com/submissions 